\title{
Behavior of Uranium(VI) during HEDPA Leaching for Aluminum Dissolution in Tank Waste Sludges
}

\author{
Brian A. Powell ${ }^{1}$, Linfeng Rao ${ }^{1}$, Kenneth L. Nash ${ }^{2}$, Leigh Martin ${ }^{2}$ \\ ${ }^{1}$ Chemical Sciences Division, Lawrence Berkeley National Laboratory, Berkeley, CA \\ ${ }^{2}$ Department of Chemistry, Washington State University, Pullman, WA
}

\begin{abstract}
Batch adsorption/dissolution experiments were conducted to examine the interactions between ${ }^{233} \mathrm{U}(\mathrm{VI})$ and a synthetic aluminum oxyhydroxide (boehmite, $\gamma$-AlOOH) in $1.0 \mathrm{M} \mathrm{NaCl}$ suspensions containing 1-hydroxyethane-1,1-diphosphonic acid (HEDPA). In the $\mathrm{pH}$ range 4 to 9, complexation of Al(III) by HEDPA significantly enhanced dissolution of boehmite. This phenomenon was especially pronounced in the neutral $\mathrm{pH}$ region where the solubility of aluminum, in the absence of complexants, is limited by the formation of sparsely soluble aluminum hydroxides. At high $\mathrm{pH}$ levels, dissolution of synthetic boehmite was inhibited by HEDPA, likely due to sorption of Al(III)/HEDPA complexes. Addition of HEDPA to equilibrated U(VI)-synthetic boehmite suspensions yielded an increase in the aqueous phase uranium concentration. The concentration of uranium continually increased over 59 days. Partitioning of uranium between the solid and aqueous phase was found to correlate well with HEDPA partitioning.
\end{abstract}

\section{INTRODUCTION}

The underground storage tanks at the Hanford site contain the byproducts from chemical separations processes for plutonium production. Over time, the material in the tanks has stratified to produce a clay-like sludge layer, a slurry phase, and an overlying salt cake. The transuranium elements are believed to be concentrated in the sludge phase, which is primarily composed of metal (oxyhydr)oxides. The current strategy for treatment of the tank waste calls for vitrification of the sludge followed by emplacement in a geological repository. The high concentration of aluminum and other non-radioactive elements increases the volume of the sludge and will in turn increase the volume of the waste package. Pretreatment of the sludge to remove non-radioactive constituents may reduce the volume of sludge. This is desirable as any reduction in volume will significantly reduce the cost of vitrification. However, the aluminum oxides and/or aluminosilicates have proven to be difficult to remove through baseline leaching processes. Previous experiments examining aluminum dissolution by treating sludge simulants with increasingly aggressive leaching procedures $\left(0.01 \mathrm{M} \mathrm{NaNO}_{2}+0.01 \mathrm{M} \mathrm{NaOH}, 3 \mathrm{M} \mathrm{NaOH}, 0.05 \mathrm{M}\right.$ glycolic acid + 0.10M NaOH, $0.10 \mathrm{M} \mathrm{HNO}_{3}, 2.0 \mathrm{M} \mathrm{HNO}_{3}, 0.5 \mathrm{M} \mathrm{HEDPA}$ ) found that no single treatment achieved complete dissolution [1]. Additionally, interactions of actinides with aluminum oxides have significant implications in designing strategies for sludge leaching.

The objective of this study was to investigate the ability of HEDPA to accomplish dissolution of synthetic boehmite and the behavior of actinides during dissolution. HEDPA is a diphosphonate complexant that forms strong complexes with actinides and aluminum in acidic to neutral and basic solutions. Reed et al. [2] performed thermodynamic studies of U(VI)-HEDPA 
systems across the $\mathrm{pH}$ range 2 to 12 and identified several $\mathrm{M}_{m} \mathrm{H}_{h} \mathrm{~L}_{l}$ complexes using potentiometry, calorimetry, and spectroscopic techniques [2]. The thermodynamic data provided by Reed et al., [2] are used below to describe observed behavior of uranium in synthetic boehmite suspensions amended with HEDPA.

\section{MATERIALS and METHODS}

The alumina used in this work was obtained from SASOL (trade name CATAPAL® B). The material was found to have the crystal structure of boehmite $(\gamma-\mathrm{AlOOH})$ determined using a Seimens D-500 Diffractometer. A surface area of $354 \mathrm{~m}^{2} \mathrm{~g}^{-1}$ was measured by $\mathrm{N}_{2}(\mathrm{~g})$ adsorption using a Micrometrics BET Surface Area Analyzer. The point-of-zero-salt-effect (PZSE) was determined to be 8.1 by potentiometric titrations in $0.01,0.1$, and $1.0 \mathrm{M} \mathrm{NaCl}$. HEDPA was obtained as a $70 \%$ aqueous solution (Sigma) and purified by recrystallization from glacial acetic acid. A stock solution of ${ }^{233} \mathrm{U}(\mathrm{VI})$ tracer was obtained from the inventory at Lawrence Berkeley National Laboratory and purified by ion exchange. Analysis by $\alpha$-spectroscopy indicated that the purified uranium contains $96.6 \%{ }^{233} \mathrm{U}$ and $3.3 \%{ }^{232} \mathrm{U}$. All other chemicals were of ACS reagent grade quality and used as received. All experiments were performed in $1 \mathrm{M} \mathrm{NaCl}$.

Working suspensions spanning the $\mathrm{pH}$ range 4 to 11 were prepared by equilibrating synthetic boehmite with $1 \mathrm{M} \mathrm{NaCl}$ for several months. These suspensions were used to prepare samples for batch experiments. The batch experiments were performed in polypropylene centrifuge tubes mixed along their longitudinal axis on an orbital shaker at $150 \mathrm{rpm}$. Batch dissolution experiments to examine the dissolution of synthetic boehmite by HEDPA were conducted in 5 $\mathrm{mM}, 10 \mathrm{mM}$, and $50 \mathrm{mM}$ HEDPA suspensions with a constant synthetic boehmite concentration of $600 \mathrm{mg} \mathrm{L}^{-1}$ (10 mM as $\mathrm{Al}$ ). Using the measured surface area and assuming a site density of 1.5 $\equiv \mathrm{AlOH}$ sites $\mathrm{nm}^{-2}$, the concentration of $\equiv \mathrm{AlOH}$ sites in $600 \mathrm{mg} \mathrm{L}^{-1}$ boehmite was approximately $0.50 \mathrm{mM}$. Therefore, the concentration of HEDPA is greater than the available number of sites, particularly in the $50 \mathrm{mM}$ HEDPA system. At specified intervals, aliquots were removed and passed through $200 \mathrm{~nm}$ nylon filters (Gelman Acrodisc). The aluminum concentration in the filtrate was measured using ICP-OES (Perkin Elmer, Optima 5300 DV). At a few intervals, the HEDPA concentration in the filtrate was measured using a spectrophotometric method [3] employing a Cary 500 Spectrophotometer.

The effect of HEDPA on uranium partitioning to synthetic boehmite was examined by equilibrating U(VI)-boehmite suspensions for 10 days and then amending the solutions with HEDPA. Preliminary kinetic tests (in the absence of HEDPA) indicated steady state uranium partitioning was achieved after 7 days. At specified intervals, aliquots were removed and passed through 30k MWCO centrifugal filters (Nanosep, approximately $12 \mathrm{~nm}$ pore size). The ${ }^{233} \mathrm{U}$ concentration in the filtrate was measured in EcoLume ${ }^{\mathrm{TM}}$ (MP Biomedicals Inc.) cocktail on a Wallac 1415 liquid scintillation counter.

\section{RESULTS and DISCUSSION}

Dissolution of synthetic boehmite with HEDPA. The aqueous phase concentration of aluminum is typically limited by the formation of sparsely soluble Al (hydr)oxide solids, especially at circum-neutral pH values. As shown in Fig. 1, addition of HEDPA clearly 

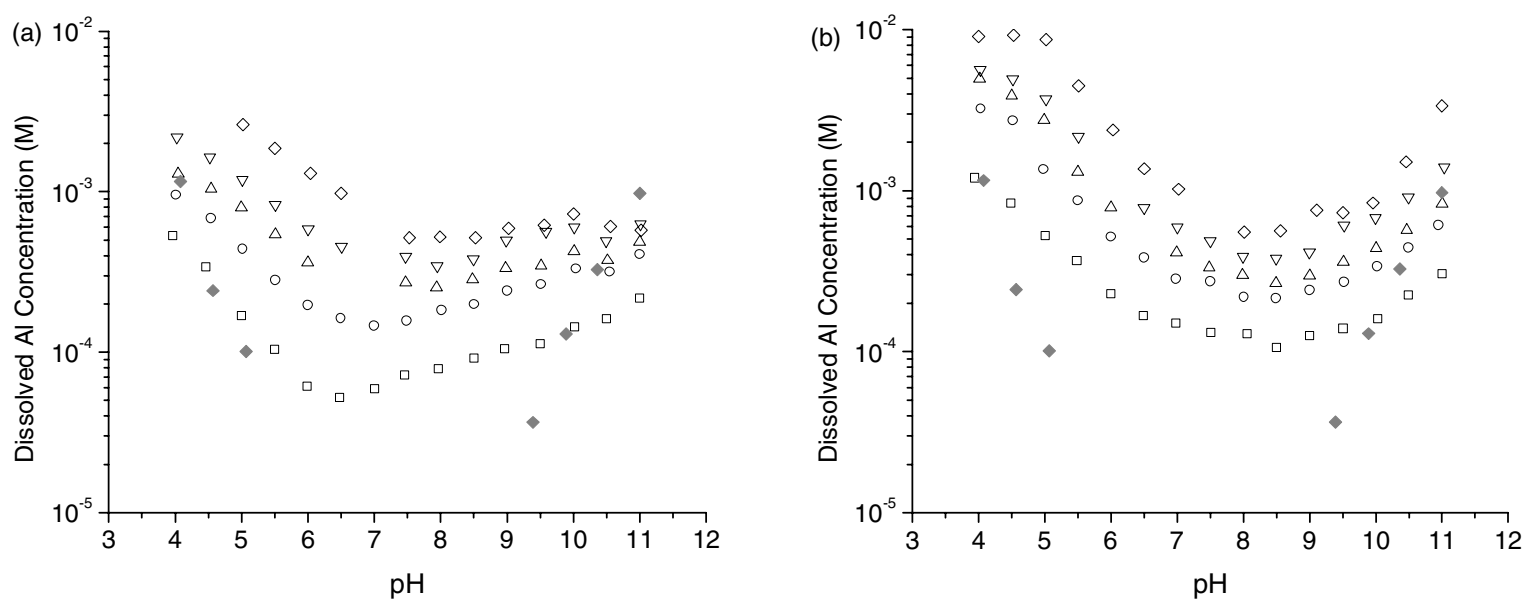

Figure 1: Effect of HEDPA on synthetic boehmite $(\gamma-\mathrm{AlOOH})$ dissolution, $[\gamma-\mathrm{AlOOH}]=600 \mathrm{mg}$ $\mathrm{L}^{-1},[\mathrm{NaCl}]=1.0 \mathrm{M}$. (a) $[\mathrm{HEDPA}]=5.4 \mathrm{mM}$; (b) $[\mathrm{HEDPA}]=50 \mathrm{mM}$. Symbols: $(\square) 2$ days, (o) 16 days, $(\Delta) 36$ days, $(\nabla) 86$ days, and $(\diamond) 135$ days. $(\diamond)$ Control system contains no HEDPA and was measured after 135 days. Error bars, typically contained within area of symbol at $95 \%$ certainty, have been removed for clarity.

enhances the dissolution of synthetic boehmite. Across the $\mathrm{pH}$ range 5 to 9 , samples amended with HEDPA have aluminum concentrations significantly higher than the control system which does not contain HEDPA. The enhanced dissolution of boehmite in the presence of HEDPA is likely the results of formation of $\mathrm{Al}_{m} \mathrm{H}_{h} \mathrm{~L}_{l}$ complexes (where L represents completely deprotonated HEDPA and $m, h$, and $l$ are stoichiometric coefficients). The exact identity of specific complexes is uncertain at this point as the data available in the current literature are not in agreement $[4,5]$. Studies of the aqueous speciation of the Al(III)-HEDPA system are underway. It was found that a 1:1 Al(III):HEDPA white precipitate formed across the neutral $\mathrm{pH}$ range [6], suggesting the low aluminum concentration across this $\mathrm{pH}$ range (Fig. 1) may be controlled by the solubility of an Al(III)/HEDPA solid phase. The Al:HEDPA molar ratio in the precipitate was measured by isolating and re-dissolving the precipitate in acid, then measuring the $\mathrm{Al}(\mathrm{III})$ and HEDPA concentrations in the resulting solution.

The concentrations of $\mathrm{Al}$ in more acidic $(\mathrm{pH} 4-5)$ and basic $(\mathrm{pH}>10)$ regions were higher than those in the neutral $\mathrm{pH}$ region (Fig. 1). At $\mathrm{pH} 4-5$, the concentrations of aluminum were comparable in systems with or without HEPDA. This is due to the formation of $\mathrm{Al}(\mathrm{OH})^{2+}$ and $\mathrm{Al}(\mathrm{OH})_{2}{ }^{+}$species yielding macromolar concentrations of aluminum. At $\mathrm{pH}>10$, the dominant aqueous species is $\mathrm{Al}(\mathrm{OH})_{4}{ }^{-}$, yielding high aluminum concentrations in systems without HEDPA. At $\mathrm{pH} 11$, the presence of HEDPA resulted in a lower aluminum concentration than that in the control system after 135 days (Fig. 1). The decrease in aluminum concentration may be the result of sorption of an Al(III)/HEDPA complex or the slow kinetics of dissociation of $\mathrm{Al}(\mathrm{III}) / \mathrm{HEDPA}$ complexes from the mineral surface.

Sorption of U(VI) to synthetic boehmite in the absence of HEDPA. Data describing the sorption of uranium in the absence of HEDPA are shown in Fig. 2 and Fig. 3. Error bars, \pm 0.03 for the fraction of U(VI) at $95 \%$ certainty, have been removed for clarity. In the absence of HEDPA, approximately $15 \%$ of the uranium was sorbed at $\mathrm{pH} 4$. As the $\mathrm{pH}$ increased, the 


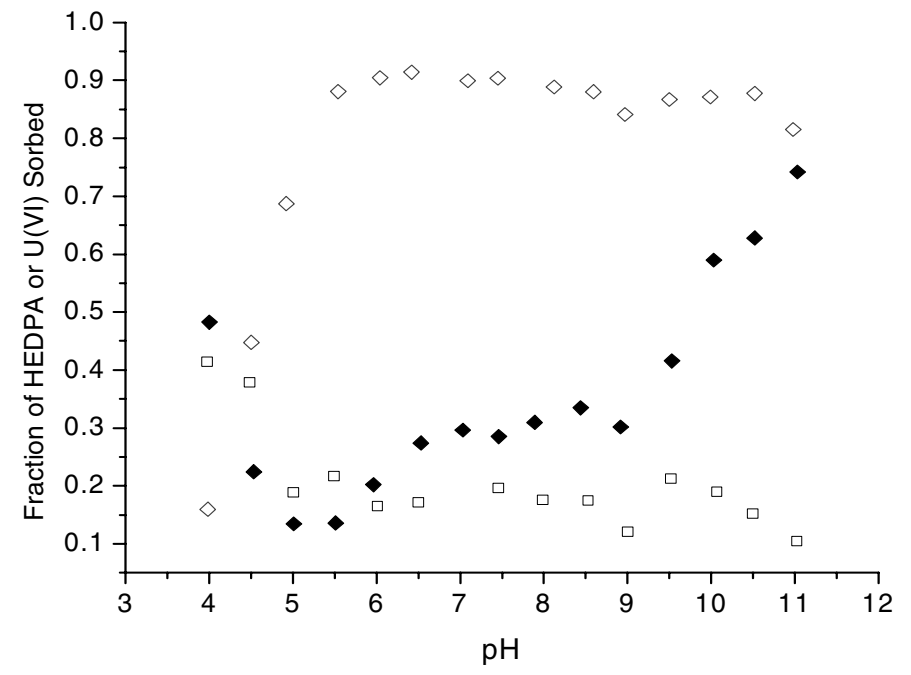

Figure. 2: Sorption of U(VI) and HEDPA to synthetic boehmite, $[\gamma-\mathrm{AlOOH}]=600 \mathrm{mg} \mathrm{L}^{-1}$. Symbols: $(\diamond)$ Sorption of U(VI) at steady state in the absence of HEDPA, initial [U(VI)] = 6.1 $\mu \mathrm{M}$; (口) Sorption of HEDPA after 72 days in the absence of uranium, [HEDPA] = $5.4 \mathrm{mM}$; $(\diamond)$ Sorption of U(VI) in $5.4 \mathrm{mM}$ HEDPA after 59 days.

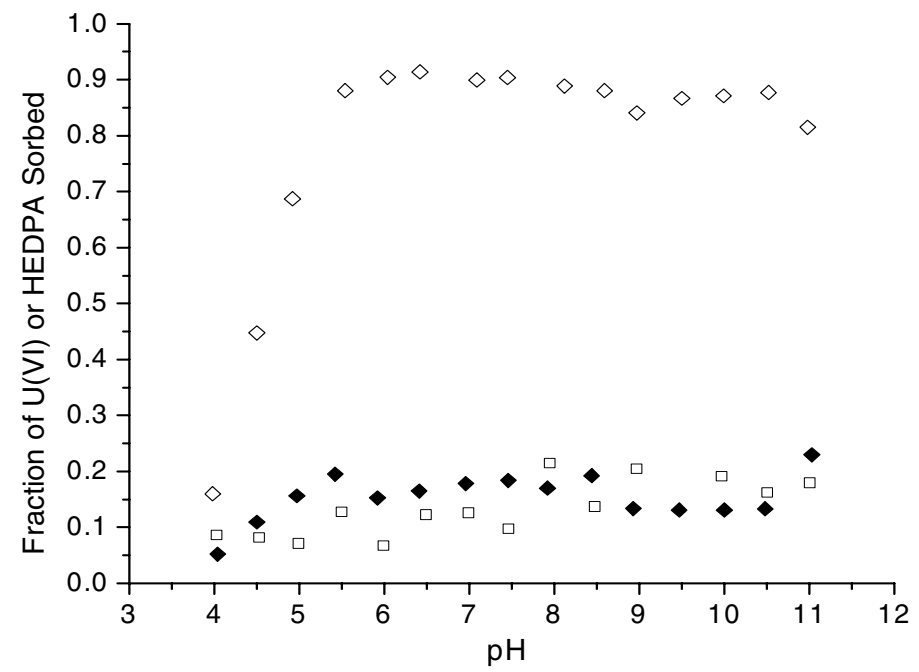

Figure 3: Sorption of U(VI) and HEDPA to synthetic boehmite, $[\gamma-\mathrm{AlOOH}]=600 \mathrm{mg} \mathrm{L}^{-1}$. Symbols: $(\diamond)$ Sorption of U(VI) at steady state in the absence of HEDPA, initial [U(VI)] $=6.1$ $\mu \mathrm{M}$; ( $\square$ ) Sorption of HEDPA after 72 days in the absence of uranium, [HEDPA] = $10.8 \mathrm{mM}$; ( ) Sorption of U(VI) in $50 \mathrm{mM}$ HEDPA after 59 days.

fraction of uranium sorbed increased until relatively steady partitioning was reached at $\mathrm{pH}>5$. Above $\mathrm{pH} 5$, approximately $90 \%$ of the total uranium was sorbed. The observed partitioning behavior could be well explained by the repulsion of the uranyl dioxocation by the positively charged surface. As the $\mathrm{pH}$ was increased, both the net positive surface charge and the positive charge on the U(VI) species decreased (the latter due to hydrolysis), resulting in stronger sorption of U(VI) onto boehmite. The cationic sorption edge around $\mathrm{pH} 5$ is consistent with observations made by Prikryl et al. [7] when examining uranium sorption to $\alpha$-alumina. 
Sorption of HEDPA to synthetic boehmite. Fig. 2 and Fig. 3 also show the fraction of HEDPA sorbed to synthetic boehmite as a function of pH. Error bars, \pm 0.04 for HEDPA at $95 \%$ certainty, have been removed for clarity. Sorption of HEDPA is likely to occur through the two phosphate groups, allowing for the use of phosphate as an analog to describe HEDPA sorption. Interactions between phosphates and metal oxide surfaces occur through ligand exchange reactions and electrostatic interactions [8]. Phosphate is capable of forming bi- and tri-dentate surface complexes and, generally, sorption of phosphate decreases as the bulk solution $\mathrm{pH}$ increases. The sorption of HEDPA shown in Fig. 2 and Fig. 3 is very similar to the sorption of phosphate. In the $5.4 \mathrm{mM}$ HEDPA systems, where the concentration of HEDPA is comparable to the surface site concentration, approximately $40 \%$ of the total HEDPA was sorbed at $\mathrm{pH}<5$ (Fig. 2). In this $\mathrm{pH}$ region, the deprotonated phosphate groups of HEDPA are attracted to the positive surface charge on the metal oxide surface and partition to the solid phase. Above $\mathrm{pH} 9.5$, there was a slight decrease in HEDPA sorption (Fig. 2). Nowack and Stone [9] observed a similar decrease in HEDPA sorption to goethite above $\mathrm{pH}$ 9. As the $\mathrm{pH}$ increased and the surface developed a net negative charge due to deprotonation of hydroxide surface sites, the anionic HEDPA ligand was repelled and partitioned to the aqueous phase. The effect of $\mathrm{pH}$ on HEDPA sorption was not as easily observed in the systems with higher HEDPA concentrations (Fig. 3). Due to the low surface site concentration relative to the HEDPA concentration, most of the HEPDA was present in the aqueous phase.

\section{Behavior of U(VI) during the dissolution of synthetic boehmite with HEDPA. Data} describing the sorption of uranium in the presence of HEDPA are also shown in Fig. 2 and Fig. 3. At $\mathrm{pH} 4$, the addition of $5.4 \mathrm{mM}$ HEDPA increased the fraction of uranium from $15 \%$ to $47 \%$ within 3 hours (the fraction remained unchanged for the remainder of the experiment up to 59 days). From $\mathrm{pH} 4$ to 5 , the fraction of uranium sorbed in $5.4 \mathrm{mM}$ HEDPA decreased from $47 \%$ to $13 \%$, which strongly correlated with the decrease of the fraction of HEDPA sorbed (Fig.2). Reed et al., [2] identified several $\mathrm{UO}_{2} \mathrm{~L}_{l} \mathrm{H}_{h}$ species in this $\mathrm{pH}$ region. The observed uranium behavior in the $5 \mathrm{mM}$ HEDPA suspension is likely the result of the formation of anionic $\mathrm{UO}_{2} \mathrm{~L}_{2} \mathrm{H}_{4}{ }^{2-}$ and $\mathrm{UO}_{2} \mathrm{~L}_{2} \mathrm{H}_{3}{ }^{3-}$ species which are attracted to the positively charged boehmite surface. Similar behavior was observed in Th(IV)- $\mathrm{PO}_{4}{ }^{3-}$-alumina systems, where addition of phosphate enhanced sorption of Th(IV) up to $\mathrm{pH} 4$ [10]. The same phenomenon was not observed in the $50 \mathrm{mM}$ HEDPA system (Fig. 3). The high concentration of HEDPA (50 mM) may have blocked all available surface sites or prevent uranium from sorbing through complex formation. In the $50 \mathrm{mM}$ HEDPA suspension, the concentration of HEDPA was 100 times the available surface site concentration and $10^{4}$ times the uranium concentration.

Across the $\mathrm{pH}$ range 6.5 to 9 , the fraction of uranium sorbed in the presence of HEDPA (5.4 $\mathrm{mM}$ and $50 \mathrm{mM}$ ) remained relatively constant (Fig. 2 and Fig. 3). The predominant species across this entire $\mathrm{pH}$ range, calculated using stability constants reported by Reed et al. [2], is $\mathrm{UO}_{2} \mathrm{~L}_{2} \mathrm{H}^{5-}$. The surface goes from a net positive to net negative charge across this $\mathrm{pH}$ range. Therefore, relatively constant uranium sorption cannot be explained solely by electrostatic interactions. As noted above, phosphate is known to form bi- and tri-dentate surfaces complexes. Therefore, sorption of uranium across this $\mathrm{pH}$ range may be the result of a $\mathrm{UO}_{2} \mathrm{~L}_{\mathrm{l}} \mathrm{H}_{\mathrm{h}}$ species coordinating the surface through a ligand exchange reaction between the phosphate groups and hydroxide surface sites. 
As the $\mathrm{pH}$ was increased from 10 to 11 , sorption of uranium increased significantly in 5.4 $\mathrm{mM}$ HEDPA and slightly in $50 \mathrm{mM}$ HEDPA systems. The significant increase of uranium sorption in $5.4 \mathrm{mM}$ HEDPA is probably due to increase in available surface sites that developed as HEDPA partitioned into the aqueous phase (Fig. 2). On the other hand, the ligand concentration in the $50 \mathrm{mM}$ HEDPA system was so high that it overwhelmed both uranium complexation and the available surface sites and HEDPA predominantly remained in solution in a wide $\mathrm{pH}$ region (4-11). As a result, only slight changes in the sorption of uranium were observed across the entire $\mathrm{pH}$ range.

\section{CONCLUSIONS}

Results from this work show that HEDPA is capable of significantly enhancing the solubility of aluminum hydroxides. The degree of enhancement depends on the concentration of HEDPA and the solution $\mathrm{pH}$. HEDPA is capable of leaching uranium from synthetic boehmite through solid phase dissolution and/or the formation of U(VI)/HEDPA complexes. Across the $\mathrm{pH}$ range examined, the speciation of U(VI)/HEDPA complexes affects the partitioning of uranium between the solid and aqueous phase. In systems where the concentration of HEDPA is low (5.4 $\mathrm{mM}$ ) and comparable to the amount of available surface sites, partitioning of uranium was observed to vary with $\mathrm{pH}$ and correlated with the partitioning of HEDPA. Experiments are continued to determine if complete dissolution occurs and provide a detailed description of the effect of HEDPA on the sorption of uranium to synthetic boehmite.

\section{ACKNOWLEDGEMENTS}

This work was supported by the Director, Office of Science, Office of Biological and Environmental Research of the U.S. Department of Energy under Contract No. DE-AC0205CH11231 at the Lawrence Berkeley National Laboratory.

\section{REFERENCES}

1. K. L. Nash, A. V. Gelis, M. P. Jensen, A. H. Bond, J. C. Sullivan, L. Rao, A. Garnov, J. Nucl. Sci. Tech., Supplement 3, 512-515 (November 2002).

2. W. Reed, et al., "Complexation of U(VI) with 1-hydroxy-1,1-ethanediphosphonic acid (HEDPA)" manuscript in preparation (2005).

3. APHA, Standard Methods for the Examination of Water and Wastewater, $17^{\text {th }}$ ed. (America Public Health Association, Washington, DC, 1989) p. 4-166.

4. S. Lacour, V. Deluchat, J. Bollinger, B. Serpaud, Talanta, 46, 999-1009 (1998).

5. E. Gumienna-Kontecka, R. Silvagni, R. Lipinski, M. Lecouvey, F. C. Marincola, G. Crisoni, V. M. Nurchi, Y. Leroux, H. Koslowski, Inorg. Chim. Acta, 339, 111-118 (2002).

6. B. A. Powell, L. Rao, unpublished data.

7. J. D. Prikryl, R. T. Pabalan, D. R. Turner, B. W. Leslie, Radiochim. Acta. 66/67, 291-296 (1994).

8. W. Stumm, The Inner-sphere Surface Complex: A Key to Understanding Surface Reactivity, ed. C. P. Huang, C.R O'Melia, and J.J. Morgan, (Advances in Chemistry Series 244, American Chemical Society, Washington, DC, 1995) pp. 1-32.

9. D. Nowack, A. T. Stone, Env. Sci. Tech. 33, 3627-3633 (1999).

10. Z. Guo, X. Yu, F. Guo, Z. Tao, J. Colloid Inter. Sci. 288, 14-20 (2005). 